\title{
ICRH for core impurity mitigation in JET-ILW
}

E. Lerche', M. Goniche, P. Jacquet, D. Van Eester, V. Bobkov, L. Colas, I. Monakhov, F. Rimini, A. Czarnecka, K. Crombé, R. Dumont, J. Hobirk, Y. Kazakov, M.-L. Mayoral, L. Meneses, J. Mlynar, C. Noble, I. Nunes, J. Ongena, V. Petrzilka, M. Reich, M. Santala, A. Shaw, M. Tsalas, and JET Contributors

Citation: AIP Conference Proceedings 1689, 030002 (2015); doi: 10.1063/1.4936467

View online: http://dx.doi.org/10.1063/1.4936467

View Table of Contents: http://aip.scitation.org/toc/apc/1689/1

Published by the American Institute of Physics 


\title{
ICRH for core impurity mitigation in JET-ILW
}

\author{
E. Lerche ${ }^{1,2, \text { a) }}$, M. Goniche ${ }^{3}$, P. Jacquet ${ }^{2}$, D. Van Eester ${ }^{1}$, V. Bobkov ${ }^{4}$, L. Colas ${ }^{3}$, \\ I. Monakhov ${ }^{2}$, F. Rimini ${ }^{2}$, A. Czarnecka ${ }^{5}$, K. Crombé ${ }^{1}$, R. Dumont ${ }^{3}$, J. Hobirk ${ }^{4}$, \\ Y. Kazakov', M.-L. Mayoral ${ }^{2}$, L. Meneses ${ }^{6}, J^{1}$ Mlynar ${ }^{7}$, C. Noble ${ }^{2}$, I. Nunes ${ }^{6}$, \\ J. Ongena ${ }^{1}$, V. Petrzilka ${ }^{7}$, M. Reich ${ }^{4}$, M. Santala ${ }^{2}$, A. Shaw ${ }^{2}$, M. Tsalas ${ }^{8}$ and \\ JET Contributors
}

\author{
EUROfusion Consortium, JET, Culham Science Centre, Abingdon, OX14 3DB, UK \\ ${ }^{1}$ LPP-ERM/KMS, Association EUROFUSION-Belgian State, TEC partner, Brussels, Belgium \\ ${ }^{2}$ CCFE Fusion Association, Culham Science Centre, Abingdon, United Kingdom \\ ${ }^{3}$ IRFM, Association EUROFUSION -CEA, Saint-Paul-Lez-Durance, France \\ ${ }^{4}$ Max-Planck-Institut für Plasmaphysik, EUROFUSION -Assoziation, Garching, Germany \\ ${ }^{5}$ IPPLM, EUROFUSION Association, Warsaw, Poland \\ ${ }^{6}$ Instituto de Plasmas e Fusão Nuclear, EUROFUSION -IST Association, Lisbon, Portugal \\ ${ }^{7}$ Institute of Plasma Physics, EUROFUSION -IPP.CR Association, Prague, Czech Republic \\ ${ }^{8}$ FOM Inst. DIFFER, EUROFUSION Association, Nieuwegein, Netherlands \\ ${ }^{a)}$ Corresponding author: ealerche@msn.com
}

\begin{abstract}
Ion cyclotron resonance frequency (ICRF) heating has been an essential component in the development of high power $\mathrm{H}$-mode scenarios in JET-ILW. The steps that were taken for the successful use of ICRF heating in terms of enhancing the power capabilities and optimizing the heating performance in view of core impurity mitigation in these experiments will be reviewed.
\end{abstract}

\section{INTRODUCTION}

Since 2011 JET is operating with a full-metal ITER-like wall (ILW), with most of the main chamber plasma facing components made from beryllium (Be) and having a tungsten (W) divertor [1]. After an initial period of divertor heat-load investigations and plasma-wall interaction studies, JET has entered a phase of high performance scenario development in a full metal environment in view of ITER [2]. In H-modes with $\mathrm{P}_{\mathrm{NBP}}>15 \mathrm{MW}$ of neutral beam injection (NBI), core $\mathrm{W}$ accumulation has become a concern since aside from degrading the plasma performance it can lead to radiative collapse of the discharges. ASDEX Upgrade [3] and more recently JET-ILW [4] experiments have shown that an efficient way of avoiding central impurity accumulation is to provide a localized heat source to the plasma centre, either by electron cyclotron (ECRH) or by ion cyclotron resonance heating (ICRH). The resulting peaked electron temperature profiles, flattened density profiles and enhanced fast ion pressure (ICRH only) have a direct impact on the transport of the high- $Z$ impurities in the plasma core, both via neo-classical and via anomalous transport effects [5-7].

JET is currently equipped with four ICRF antennas with a nominal generated power capability of 12MW in ELM tolerant configuration [8]. The actual fraction of the generated RF power effectively coupled to the plasma is lower and depends on the antenna-plasma coupling that, for given RF settings, is dictated by the scrape-off-layer (SOL) density profile in front of the antennas and the pedestal properties. Both are usually unfavourable in high power Hmode discharges characterized by low SOL density and steep gradients. The absorption of the waves in the plasma,

* See the Appendix of F. Romanelli et al., Proc. of the $25^{\text {th }}$ IAEA Fusion Energy Conference, 2014, St. Petersburg, Russia

Radio Frequency Power in Plasmas

AIP Conf. Proc. 1689, 030002-1-030002-8; doi: 10.1063/1.4936467

2015 AIP Publishing LLC 978-0-7354-1336-8/\$30.00 
on the other hand, is mainly influenced by the core plasma profiles and by the choice of the minority ion species and its concentration. The large temperatures and densities typical of H-modes are beneficial for the RF wave absorption but the effective core electron heating still depends on the slowing-down characteristics of the minority ions and is thus influenced by their concentration and the amount of RF power absorbed in the plasma.

The main results concerning the optimization of ICRH for core impurity control in JET-ILW H-modes will be summarized. The first step was to increase the power capability of the RF system, by optimizing the RF plant operation / antenna conditioning and by enhancing the antenna-plasma coupling using localized gas injection (section 2). The second step consisted in optimizing the RF power absorption and core electron heating by finetuning the IC resonance position and the minority concentration (section 3). Some examples of core impurity mitigation with ICRH in high power baseline $\mathrm{H}$-modes are given afterwards. The article ends with a brief summary and prospects.

\section{RF COUPLING OPTIMIZATION}

The first step to improve the application of ICRF in high NBI power H-modes was to optimize the RF antennaplasma coupling to increase the ICRF power capability and reliability in these conditions. This was achieved by tailoring the far-SOL density using dedicated gas injection modules (GIMs) located in the main vacuum chamber, as opposed to the default divertor injection usually adopted $[9,10]$. The results are summarized in Fig.1, where the inter-ELM coupling resistances of the four ICRF antennas are plotted as function of the gas injection rate $\left(\mathrm{D}_{2}\right)$ for different fuelling locations (antennas $\mathrm{C}$ and D have the same resistance in ELM-tolerant configuration). Although some changes in the SOL properties were observed, the core and pedestal plasma parameters were not significantly affected by switching the fuelling location in the studied gas injection range [10].
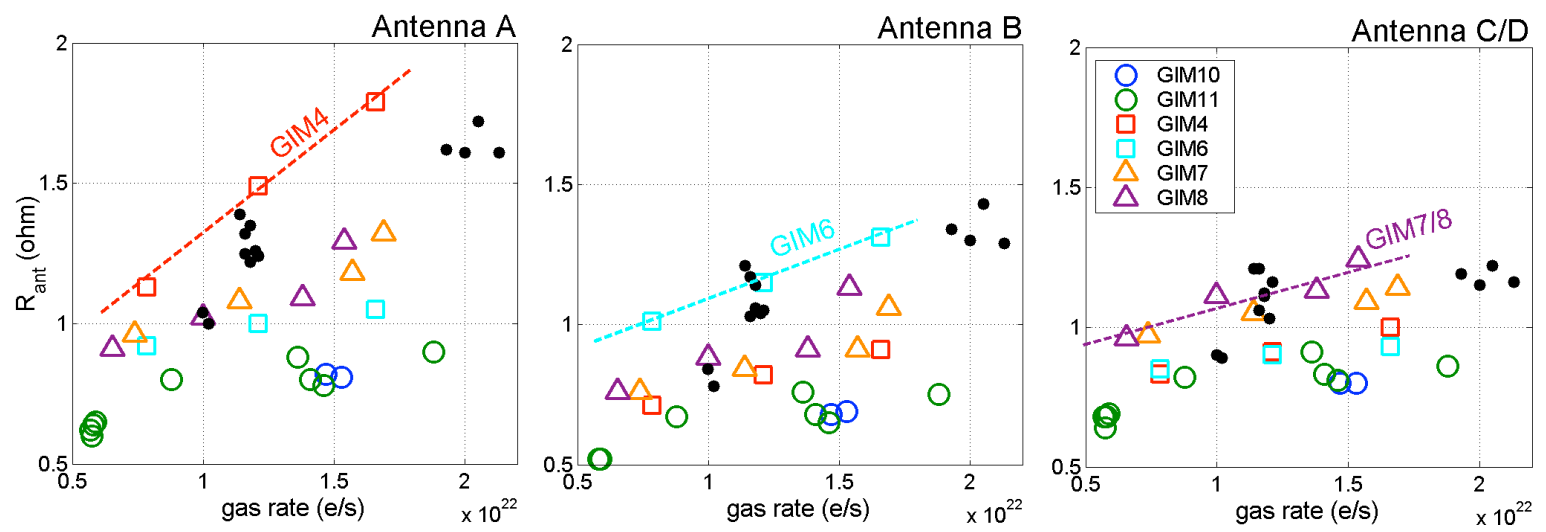

FIGURE 1. Coupling resistances of the ICRF antennas as function of the gas injection rate for different fuelling locations:

Divertor (circles), mid-plane (squares) and top injection (triangles). The data represent 1s averages of the inter-ELM RF signals at constant gas rate.

The results show that the strongest coupling enhancement is obtained with mid-plane fuelling close to the antennas (GIM4 is $\sim 1 \mathrm{~m}$ from antenna A, GIM6 $\sim 2 \mathrm{~m}$ from antenna B). Top injection (GIMs 7, 8) has a somewhat weaker impact but it affects all antennas in a similar way, since it leads to a toroidally homogeneous rather than local enhancement of the far-SOL density [9]. Divertor fuelling (GIMs 10,11) has the lowest impact on the antennaplasma coupling and its effect is barely sensitive to the amount of gas injected. By using a combination of mid-plane and top gas fuelling (black dots on Fig. 1), the coupling resistance of all ICRF antennas could be simultaneously maximized and up to $6.5 \mathrm{MW}$ of ICRF power was reliably coupled to high power H-mode plasmas. Preliminary investigations indicate that main chamber fuelling is also beneficial for minimizing the RF-induced plasma-wall interaction produced by RF sheath effects [10]. 


\section{ICRF HEATING OPTIMIZATION}

For given plasma parameters (temperature, density, magnetic field, etc..), the ICRF heating can be optimized by fine-tuning the position of the IC resonance layer and by adjusting the minority (hydrogen) concentration. The influence of both parameters on the ICRH performance in JET-ILW deuterium H-modes was studied.

The ICRF generators in JET only operate at a discrete set of frequencies. Therefore, fine tuning of the IC resonance position in the plasma has to be done by adjusting the toroidal magnetic field for a given RF frequency. Although it is well known that on-axis ICRF heating has a much stronger impact on the central plasma temperature than off-axis heating, it is not yet clear how central the resonance has to be for impurity control to be achieved. For example, in plasmas with sawtooth activity it is possible that it is sufficient for the ICRF absorption (heat source) to be located inside the $\mathrm{q}=1$ surface for efficient core heating to take place due to the fast energy redistribution inside $\mathrm{q}=1$ after a sawteeth crash. The influence of the IC absorption position on various core plasma parameters is depicted in Fig. 2. The data represent $0.3 \mathrm{~s}$ time averaged values in pulses with $\mathrm{B}_{0}$ scans $\left(2.6 \mathrm{~T}<\mathrm{B}_{0}<3 \mathrm{~T}\right)$ and otherwise similar conditions: $\mathrm{P}_{\mathrm{nbi}}=15-18 \mathrm{MW}, \mathrm{P}_{\mathrm{icrf}}=4 \mathrm{MW}, \mathrm{n}_{\mathrm{e} 0}=7.5 \pm 0.5 \times 10^{19} / \mathrm{m}^{3}$. In all cases the magnetic axis is at $\mathrm{R}_{0}=3.0 \mathrm{~m}$ and the $\mathrm{q}=1$ flux surface is around $\mathrm{R}-\mathrm{R}_{0}=0.20-0.25 \mathrm{~m}$. The normalization of the central SXR radiation with $\mathrm{T}_{\mathrm{e} 0}$ used here as a proxy for the impurity content is justified at high temperatures $\left(T_{e}>3-4 k e V\right)$, where high-Z impurity line emission is assumed to be the dominant contribution to the SXR radiation and the cooling factor is roughly proportional to $\mathrm{T}_{\mathrm{e}}[11]$.
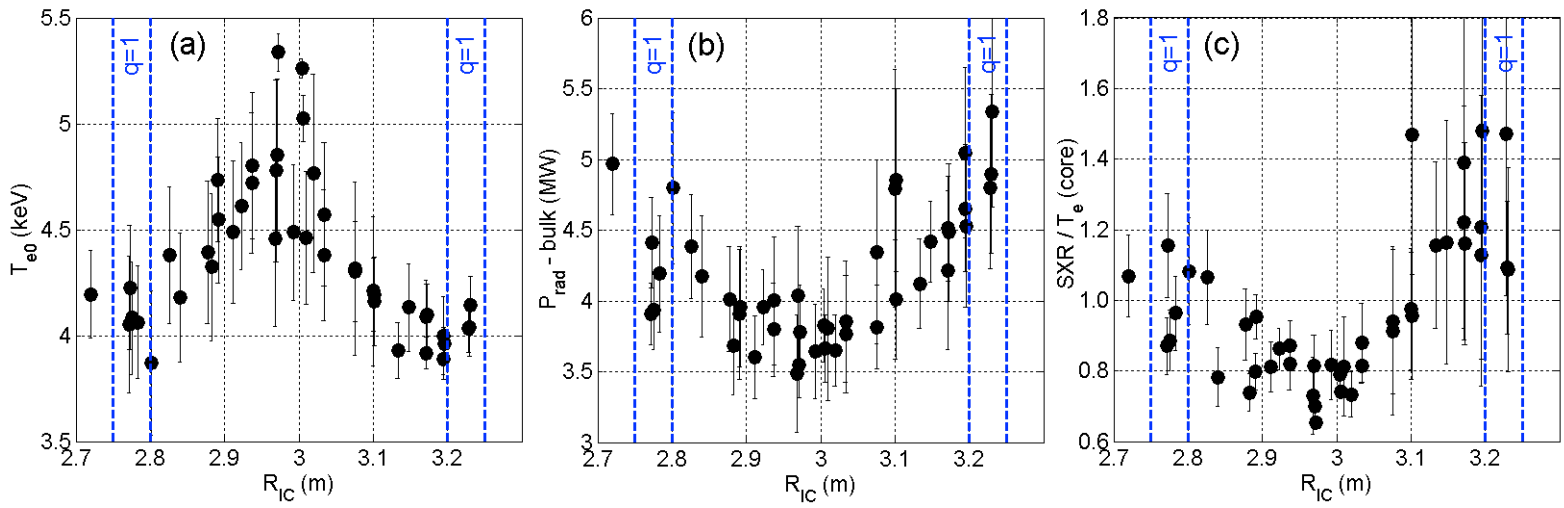

FIGURE 2. (a) Central electron temperature (ECE), (b) bulk radiated power (bolometer) and (c) central SXR emission (normalized to $\mathrm{T}_{\mathrm{e} 0}$ ) as function of the ion-cyclotron resonance position in a series of similar $\mathrm{H}$-mode discharges with $15 \mathrm{MW}<\mathrm{P}_{\text {nbi }}<18 \mathrm{MW}$ and $\mathrm{P}_{\text {icrf }}=4 \mathrm{MW}$ at $\mathrm{f}=42 \mathrm{MHz}$.

The importance of applying ICRH near the plasma centre is clear: The central electron temperature (a) peaks and both the bulk radiation measured by bolometry (b) and the core impurity emission (c) are minimized when the IC resonance is located within $\pm 10 \mathrm{~cm}$ from the magnetic axis $\left(2.9 \mathrm{~m}<\mathrm{R}_{\mathrm{IC}}<3.1 \mathrm{~m}\right)$. The bulk radiated power and the normalized SXR radiation in the plasma core are reduced by $\sim 30 \%$ when central rather than off-axis ICRH is applied, indicating more efficient impurity screening in these conditions. The strong variation of all quantities with $\mathrm{R}_{\mathrm{IC}}$ in the region $\mathrm{q}<1$ suggests that it is not sufficient to heat the plasma inside the $\mathrm{q}=1$ surface for impurity mitigation but rather apply the ICRF power more centrally. However, these results are partly biased by the fact that the sawteeth periods are influenced by the location of the ion-cyclotron resonance w.r.t. the $\mathrm{q}=1$ surface and dedicated studies aiming at simultaneously control the sawteeth and provide core ICRF heating are ongoing [12].

The minority concentration plays two different roles in the fundamental ICRF heating scheme: (i) The heating efficiency (related to the single-pass absorption of the waves) is influenced by the minority concentration, since the latter impacts on the plasma's dielectric response and thus on the RF field polarization and power absorption of the waves in the plasma; (ii) The slowing-down of the minority ions switches from electrons to ions when the minority population is increased, because the fast ion tails created by ICRH are reduced. The impact of the $\mathrm{H}$ concentration on the ICRF performance in JET-ILW H-modes was reported in [13] and a summary is given in Fig. 3, where various quantities are plotted as function of $\mathrm{X}[\mathrm{H}]=\mathrm{n}_{\mathrm{H}} / \mathrm{n}_{\mathrm{e}}$. The data on the last two graphs correspond to $0.3 \mathrm{~s}$ averages of the various quantities taken in similar discharges with $\mathrm{B}_{0}=2.7 \mathrm{~T}, \mathrm{I}_{\mathrm{P}}=2.5 \mathrm{MA}, \mathrm{P}_{\text {icrf }}=5 \mathrm{MW}$ at $\mathrm{f}=42.5 \mathrm{MHz}$, $\mathrm{P}_{\mathrm{nbi}}=15 \mathrm{MW}$ and the plasma density was constant around $\mathrm{n}_{\mathrm{e} 0}=(7.0 \pm 0.5) \times 10^{19} / \mathrm{m}^{3}$. 

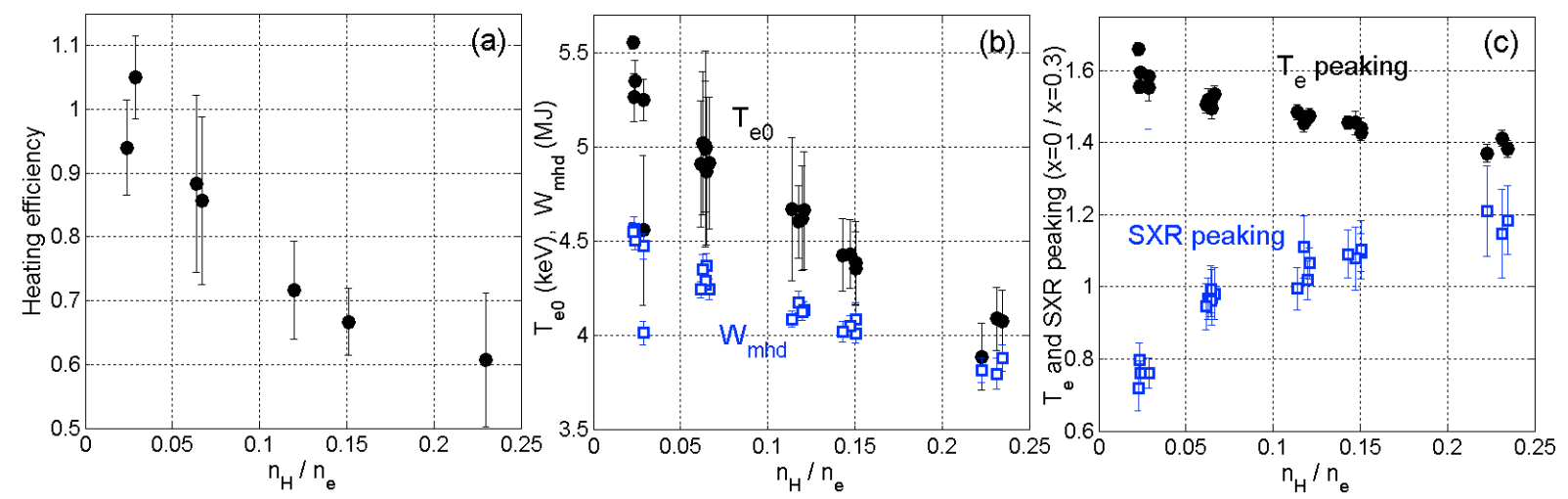

FIGURE 3. (a) ICRF heating efficiency estimated from break-in-slope analysis of the plasma stored energy as function of $\mathrm{X}[\mathrm{H}]$;

(b) Central plasma temperature from ECE (circles) and plasma stored energy (squares) as function of X[H]; (c) Electron temperature peaking from $\mathrm{ECE}$ at $\mathrm{r} / \mathrm{a}=0$ and $\mathrm{r} / \mathrm{a}=0.3$ (circles) and impurity peaking from $\mathrm{SXR}$ measurements (squares) as function of $\mathrm{X}[\mathrm{H}]$. The latter was estimated by the ratio between the line integrated data from vertical SXR channels crossing the mid-plane at $\mathrm{r} / \mathrm{a}=0$ and $\mathrm{r} / \mathrm{a}=0.3$ corrected by the local temperatures.

The heating efficiency (a) decreases from about 0.9-1.0 at low $\mathrm{H}$ concentrations to approximately 0.6 at $\mathrm{X}[\mathrm{H}]=0.25$ and is about $10-20 \%$ higher than what was observed in L-mode in earlier JET-ILW experiments [14] for all $\mathrm{X}[\mathrm{H}]$ values. The main difference is that in the $\mathrm{H}$-mode discharges (higher densities) the heating efficiency remains high at low minority concentrations $\mathrm{X}[\mathrm{H}]<0.05$, an operational window that is attractive for electron heating from the collisional (slowing-down) point of view and is not available in L-mode. Strong sawteeth stabilization was observed in these conditions but neither deleterious MHD activity nor significant impurity accumulation were an issue in the studied discharges (the electron temperature remains sufficiently peaked). The fact that the central electron temperature decreases faster than the plasma stored energy (b) when $\mathrm{X}[\mathrm{H}]$ is increased is expected, since the latter is mainly proportional to the RF heating efficiency while the central temperature also depends on the slowing-down characteristics of the fast ions. NPA measurements indicate that, opposite to what was observed in Lmode, a decrease of the number of fast ions in the energy range $100 \mathrm{keV}<\mathrm{E}<600 \mathrm{keV}$ with $\mathrm{X}[\mathrm{H}]$ rather than a decrease in the ion tail effective temperature is the main responsible for this effect at large plasma densities. As a consequence, the electron temperature profile becomes less peaked at high $\mathrm{H}$ concentrations and the core impurity screening is less efficient, as depicted in Fig. 3c.

\section{EXAMPLE OF ICRH ASSISTED H-MODES}

A comparison between a NBI-only and an ICRF assisted baseline H-mode discharge with $\mathrm{B}_{0}=2.7 \mathrm{~T} / \mathrm{I}_{\mathrm{P}}=2.5 \mathrm{MA}$ and similar auxiliary input power $(19 \mathrm{MW})$ is shown in Fig. 4. In the NBI+ICRF discharge $\left(\mathrm{P}_{\text {icrf }}=5-6 \mathrm{MW}\right)$, the central electron temperature (c) is about $1.5-2 \mathrm{keV}$ higher than in the NBI-only pulse despite the comparable plasma stored energies (b). Although there is no gradual impurity accumulation in this NBI-only example, the inter-sawteeth impurity peaking is clearly much stronger than in the NBI+ICRF case as seen by the large increase in the core SXR emission (d) as compared to the off-axis one (e) and the $\mathrm{T}_{\mathrm{e} 0}$ saturation during the sawteeth evolution (c). In the $\mathrm{NBI}+\mathrm{ICRF}$ pulse the central SXR emission is less sensitive to the sawteeth oscillations despite their larger amplitude, indicating the absence of heavy impurities in the plasma core, but the off-axis emission as well as the total bulk radiation is somewhat higher. 

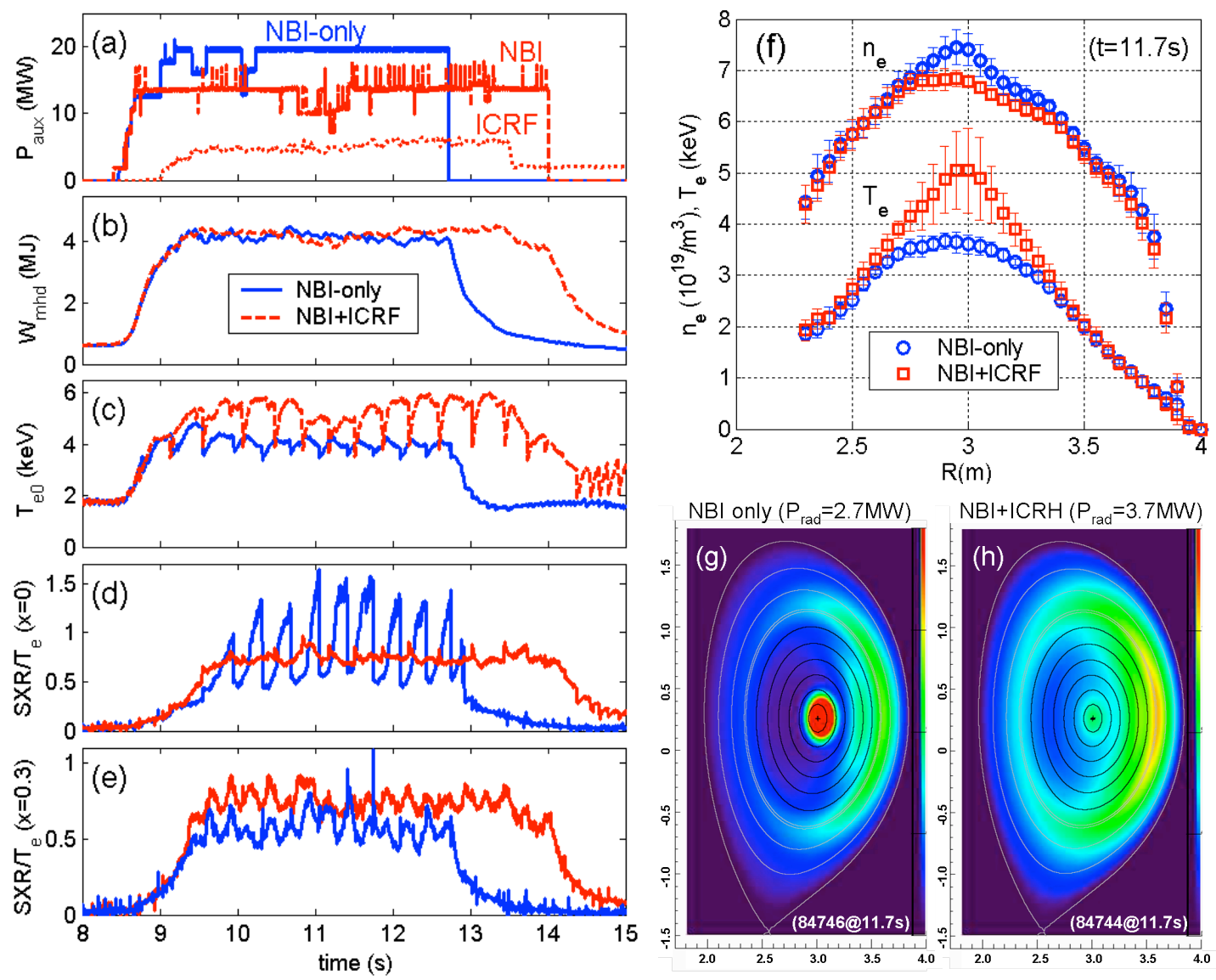

FIGURE 4. Comparison of two similar discharges with NBI-only (JPN 84744) and NBI+ICRF (JPN 84746) with the same auxiliary power input: (a) NBI and ICRF power; (b) stored plasma energy; (c) central electron temperature (ECE); (d,e) core and off-axis SXR emission (normalized to $\mathrm{T}_{\mathrm{e}}$ ); (f) electron density and temperature profiles (LIDAR) and (g,h) 2D radiation profiles $(\mathrm{SXR})$ at $\mathrm{t}=11.7 \mathrm{~s}$.

The less peaked impurity profiles seen in ICRF assisted discharges are mainly a consequence of the higher $T_{e}$ peaking and lower $n_{e}$ peaking (f) observed in this case, which for $T_{e}=T_{i}$ are consistent with an outward neoclassical convection of high-Z impurities near the plasma centre [15]. This is corroborated by dedicated impurity transport studies using $\mathrm{W}$ and Mo laser blow-off injection [16]. Theoretical studies suggest that collisions between the W ions and the RF-accelerated ions with moderate energies also contribute to the observed core impurity screening in this case [17]. The impact of ICRF heating on the spatial redistribution of the high- $Z$ impurities in the plasma is illustrated by the tomographic reconstruction of the soft X-ray radiation (g,h) for the two discharges discussed. In both cases most of the impurities are concentrated around mid-radius at the low-field-side of the plasma but in the NBI-only case a strong and localized SXR emission is observed in the very plasma core, confirming that central impurity accumulation is occurring. In the ICRF assisted discharge, the central emission is largely suppressed but the radiation at mid-radius is somewhat enhanced. The fact that the total radiation is higher in the NBI+ICRF case indicates that on top of the high- $Z$ impurity redistribution (from on- to off-axis), additional impurity sources are present when ICRF is applied. This highlights the importance of mitigating the RF-induced plasma-wall interaction for efficient impurity control with ICRH in full-metal tokamaks.

Dedicated studies of a large dataset of JET-ILW baseline H-modes reveal that the ability to control impurity accumulation with ICRH is not directly related to the amount of ICRF power coupled to the plasma, but to the impact it has on the central kinetic profiles, in particular on the $T_{e}$ peaking (which to some extent also reflects the fast particle screening effect). For example, higher density discharges require more ICRF power to reach similar $T_{e}$ peaking than low density ones since the slowing-down time of the fast ions decreases with the background density and a larger fraction of the absorbed RF power is channelled to the bulk ions rather than to the electrons. Fig. 5 
summarizes the effect of ICRH on the electron temperature and density peaking (a) and its consequences on core impurity peaking (b) in a series of similar high power $\mathrm{H}$-modes with $\mathrm{B}_{0}=2.7 \mathrm{~T}, \mathrm{I}_{\mathrm{P}}=2.5 \mathrm{MA}, \mathrm{P}_{\mathrm{NBI}}>15 \mathrm{MW}$ and $\mathrm{n}_{\mathrm{e} 0}=7$ $8 \times 10^{19} / \mathrm{m}^{3}$. The changes in the electron density peaking (hollow) with ICRF power are small in the analyzed dataset but the temperature peaking (full) increases considerably with $\mathrm{P}_{\text {ICRF }}$, reaching $20-30 \%$ higher values than in NBIonly pulses when $6 \mathrm{MW}$ of ICRF power is applied. The impurity peaking (b) decreases strongly with the electron temperature peaking and reaches unity when $\mathrm{T}_{\mathrm{e}}(\mathrm{r} / \mathrm{a}=0) / \mathrm{T}_{\mathrm{e}}(\mathrm{r} / \mathrm{a}=0.3)>1.5$, which in these discharges is achieved with $\mathrm{P}_{\mathrm{ICRF}}>4 \mathrm{MW}$ coupled to the plasma.
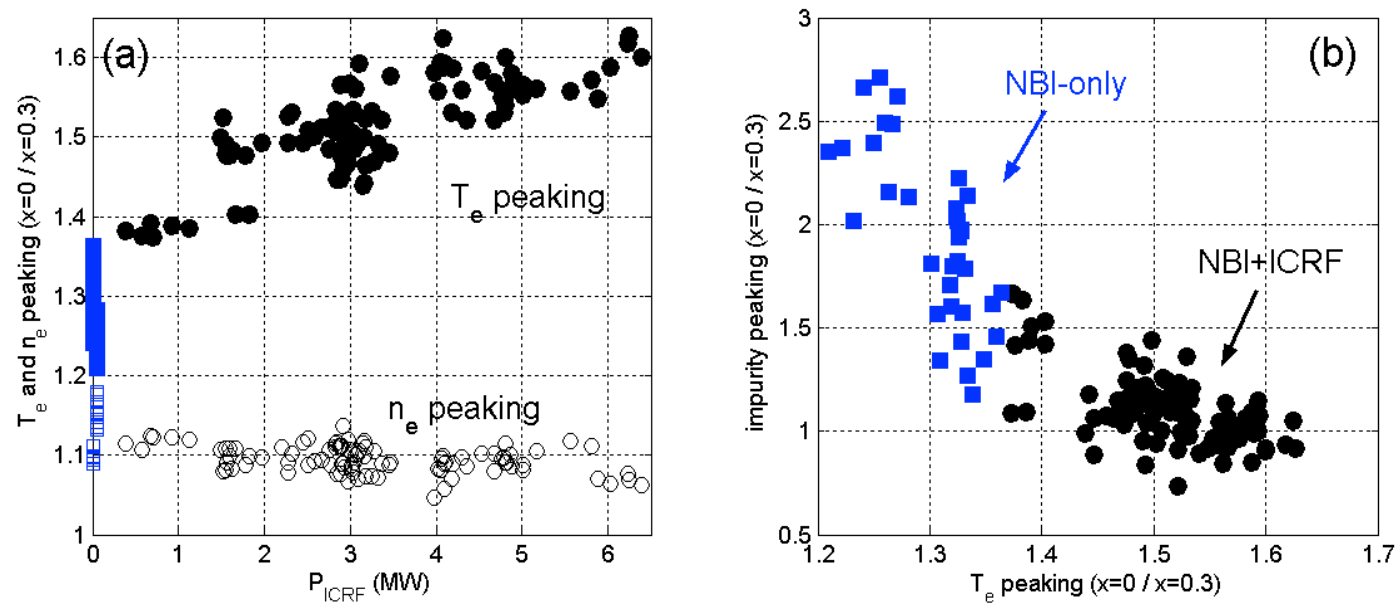

FIGURE 5. (a) Electron temperature peaking from ECE data and density peaking from LIDAR (ratio at $r / a=0$ and $r / a=0.3$ ) as function of the ICRF power; (b) impurity peaking from soft X-ray measurements (corrected with the local temperatures) as function of the $\mathrm{T}_{\mathrm{e}}$ peaking.

In high confinement discharges ICRF heating plays an even more important role on the plasma stationarity and on the safe H-L back-transition and discharge landing. In particular, high power $\mathrm{N}_{2}$ seeded H-modes could only be sustained when sufficient amount of ICRH was applied in the discharge [18]. This is illustrated in Fig. 6, where a NBI-only and an ICRF assisted high triangularity $\mathrm{N}_{2}$-seeded discharge with $\mathrm{B}_{0}=2.7 \mathrm{~T} / \mathrm{I}_{\mathrm{P}}=2.5 \mathrm{MA}$ and similar auxiliary input power $(20 \mathrm{MW})$ are compared. The same amount of $\mathrm{N}_{2}(\sim 1 \%)$ was injected in the two cases at $\mathrm{t}=10 \mathrm{~s}$. 

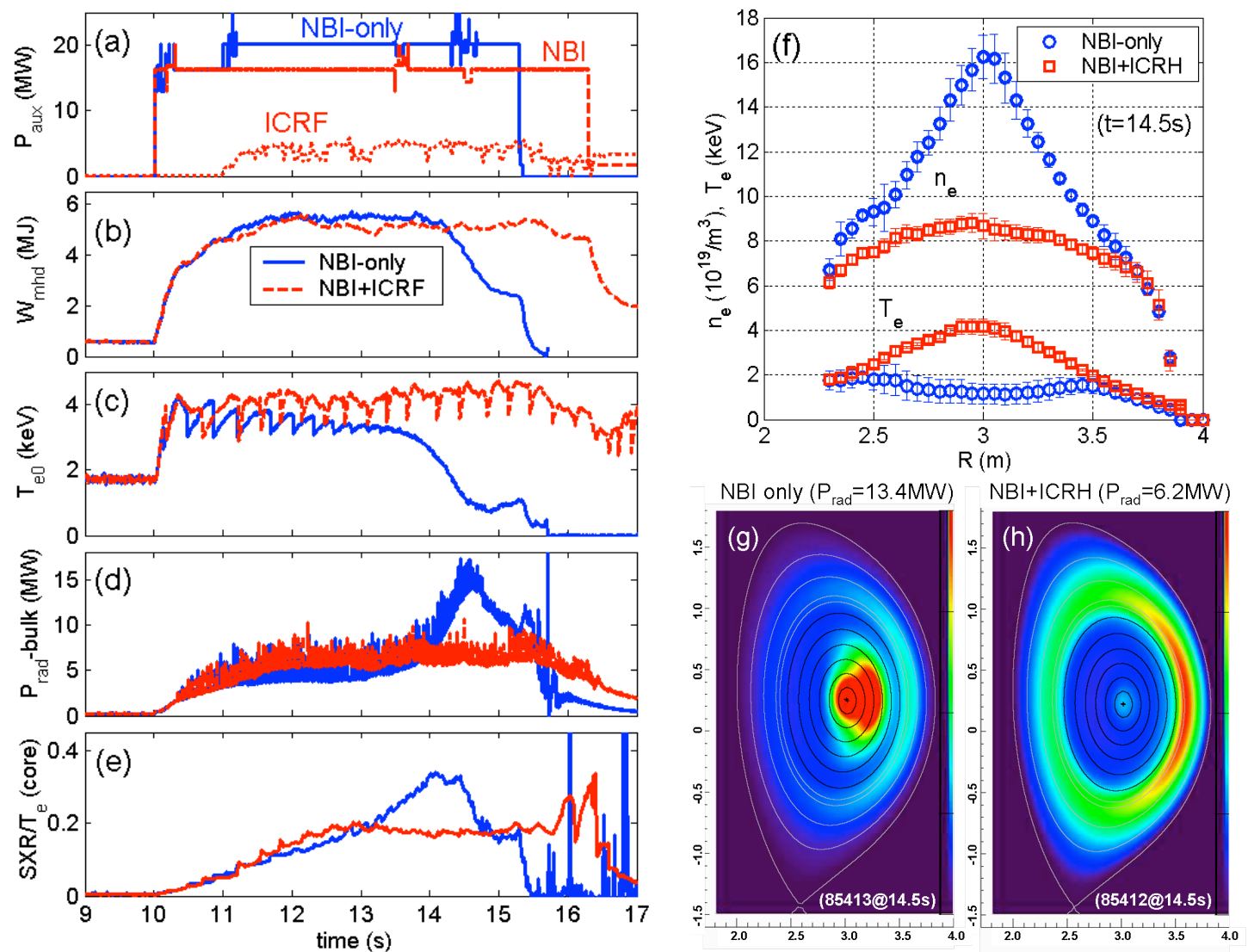

FIGURE 6. Comparison of two similar high- $\delta \mathrm{N}_{2}$ seeded discharges with NBI-only (JPN 85413) and NBI+ICRF (JPN 85412) with comparable auxiliary power input: (a) NBI and ICRF power; (b) stored plasma energy; (c) central electron temperature (ECE); (d) bulk radiated power (bolometry), (e) core SXR emission normalized to $\mathrm{T}_{\mathrm{e} 0}$; (f) electron density and temperature profiles (LIDAR) and (g,h) 2D radiation profiles (SXR) at $\mathrm{t}=11.7 \mathrm{~s}$.

In this example, the NBI-only discharge suffers from slow impurity accumulation as seen by the gradual increase of the core SXR emission (e) throughout the pulse and the systematic decay of the central electron temperature (c), including the eventual suppression of sawteeth at $t \approx 13 \mathrm{~s}$. At approximately the same time, the bulk radiation (d) starts to increase and at $\approx \approx 14 \mathrm{~s}$ it shoots-up rapidly leading to a radiative collapse of the discharge followed by a disruption. The central plasma density (not shown) also increases gradually during the NBI-only pulse from $\mathrm{n}_{\mathrm{e} 0} \approx 1 \times 10^{20} / \mathrm{m}^{3}$ (at $\mathrm{t} \approx 11 \mathrm{~s})$ to $\mathrm{n}_{\mathrm{e} 0} \approx 1.2 \times 10^{20} / \mathrm{m}^{3}$ at $\mathrm{t}=13 \mathrm{~s}$ and starts to strongly peak afterwards. In the ICRH assisted discharge, on the other hand, $5 \mathrm{MW}$ of ICRH power is enough to keep the temperature and density peaking constant during the entire $\mathrm{H}$-mode flat-top at levels that are favourable for neoclassical impurity screening, allowing to reach a stationary discharge despite of the high levels of central SXR emission and bulk plasma radiation characteristic of seeded plasmas. At $\mathrm{t}>14 \mathrm{~s}$, the kinetic profiles of the NBI-only discharge are completely different from the NBI+ICRH case (f): The central electron density reaches $n_{\mathrm{e} 0}=1.6 \times 10^{19} / \mathrm{m}^{3}$ and the density profile is extremely peaked while the electron temperature has become hollow with central values below $\mathrm{T}_{\mathrm{e} 0}<2 \mathrm{keV}$ and there is a strong accumulation of heavy impurities in the plasma centre, as seen from the massive core radiation measured in the NBI-only discharge (g). In the NBI+ICRF case, the kinetic profiles remain roughly constant throughout the pulse, indicating that the heavy impurities have been screened from the plasma core and remain in their original mid-radius position (h). 


\section{SUMMARY}

ICRF heating is being regularly used in high power JET-ILW H-mode discharges to avoid impurity peaking and to assist on the $\mathrm{H}-\mathrm{L}$ transition and plasma landing. By using optimized gas injection favourable for RF antennaplasma coupling based on distributed main chamber fuelling, $\mathrm{P}_{\mathrm{ICRF}}=4-6 \mathrm{MW}$ was routinely coupled to high NBI power $\mathrm{H}$-modes, the exact value depending on the plasma configuration and on the gas level used. The ICRF heating was optimized in terms of the ion cyclotron resonance position and the minority (hydrogen) concentration. It was seen that central ICRH and low minority concentrations give the best results for electron heating with absorption efficiencies up to $90-100 \%$, leading to sufficiently peaked electron temperature profiles (and relatively flat density profiles) for high- $\mathrm{Z}$ impurity screening in most scenarios. The study of a large dataset of 2.5MA baseline H-modes revealed that for efficient core impurity mitigation it is vital to sustain sufficiently peaked plasma temperatures to favour outward neoclassical convection, but other mechanisms such as fast ion collisional screening may also be important. In high density regimes such as $\mathrm{N}_{2}$-seeded $\mathrm{H}$-modes, stationary plasmas could only be achieved when sufficient amount of ICRH was applied early enough in the discharge. In these cases the density flattening induced by ICRH also plays a significant role on the impurity screening.

Preliminary studies of high confinement hybrid discharges and fully developed ( $\left.\mathrm{I}_{\mathrm{P}}>3 \mathrm{MW}\right)$ baseline H-modes in preparation for DT operation in JET-ILW suggest that 5MW of ICRF power is marginal for reliable impurity control but the RF heating optimization in these conditions is still ongoing. In parallel, further improvement of the ICRF system as well as the reinstallation of the ITER-like antenna [19] are planned for the next experimental campaigns, and are expected to provide sufficient amount of ICRH power for a successful exploitation of these scenarios. The results presented suggest that ICRH can also contribute to high-Z impurity control in ITER, in addition to its traditional role of bulk plasma heating for enhancing the fusion reactions.

\section{ACKNOWLEDGEMENTS}

This work has been carried out within the framework of the EUROfusion Consortium and has received funding from the EURATOM research and training programme 2014-2018 under grant agreement No 633053. The views and opinions expressed herein do not necessarily reflect those of the European Commission.

\section{REFERENCES}

1. G. Matthews et al., Phys. Scr. 2011 (2011) 014001

2. I. Nunes et al., IAEA 2014 (St. Petersburg)

3. R. Neu et al., Plasma Phys. Control. Fusion 49 12B (2007) B59-B70

4. M. Goniche et al., EPS 2014 (Berlin)

5. M. Valisa et al., Nucl. Fusion 51 (2011) 033002

6. R. Dux et al., IAEA 2010 (Daejon)

7. C. Angioni et al., Phys. Plasmas 14 (2007) 055905.

8. M. Graham et al., Plasma Phys. Control. Fusion 54 (2012) 074011.

9. E. Lerche et al., Journal of Nucl. Materials, 21st PSI (2014) (in press)

10. P. Jacquet et al., IAEA 2014 (St. Petersburg)

11. T. Puetterich et al., IAEA 2012 (San Diego)

12. J. Graves et al., EPS 2014 (Berlin)

13. D. Van Eester et al., EPS 2014 (Berlin)

14. E. Lerche et al., Nucl. Fusion 54 (2014) 073006

15. S.P. Hirshman and D.J. Sigmar, Nucl. Fusion 21 (1981) 1079

16. M. Valisa et al., IAEA 2014 (St. Petersburg)

17. F. Casson et al., Plasma Phys. Control. Fusion 57 (2015) 014031

18. C. Giroud et al., IAEA 2014 (St. Petersburg)

19. F. Durodié et al., Plasma Phys. Control. Fusion (2012) 54074012 